\title{
CONTRAST AND COLOR BALANCE ENHANCEMENT FOR NON-UNIFORM ILLUMINATION RETINAL IMAGES
}

\author{
Preecha VONGHIRANDECHA, Montri KARNJANADECHA, Sathit INTAJAG
}

\begin{abstract}
Color retinal images play an important role in supporting a medical diagnosis. However, some retinal images are unsuitable for diagnosis due to the non-uniform illumination. In order to solve this problem, we propose a method for improving non-uniform illumination that can enhance the image quality of a color fundus photograph suitable for reliable visual diagnosis. Firstly, a hidden anatomical structure in dark regions of the retinal images is revealed by improving the image luminosity with gamma correction. Secondly, multi-scale tone manipulation is then used to adjust the image contrast in the lightness channel of $L^{*} a^{*} b^{*}$ color space. Finally, color balance is adjusted by specifying the image brightness based on Hubbard's specification. The performance of the applied method has been evaluated against the data from the DIARETDB1 dataset. The results obtained show that the proposed algorithm performs well for correcting the non-uniform illumination of color retinal images.
\end{abstract}

Keywords: human visual perception; image contrast and color balance enhancement; non-uniform illumination; retinal image

\section{INTRODUCTION}

According to the World Health Organization, eye diseases such as age-related macular degeneration (AMD), cataract, and glaucoma are the main cause of blindness in the elderly worldwide $[1,2]$. Most current diagnostic systems are based on color retinal photography. However, the image may be unsatisfactory for diagnosis due to their low quality caused by camera properties, non-uniform illumination, and the experience of photographers [3]. Consequently, image enhancement algorithms are widely used to improve the image quality to provide better visibility of retinal anatomical structures.

Histogram equalization (HE) is a common contrast enhancement technique which utilizes the cumulative distribution function (CDF) of the input image for stretching the dynamic range of intensity levels. The main drawback of $\mathrm{HE}$ is that it tends to shift the mean intensity value to the middle of the dynamic range. In some cases, HE provides the results over enhancement or unnatural appearance. To overcome the mentioned drawback, various methods including BPHEME [4], BPDHE [5], and BPDFHE [6] are proposed to preserve image brightness. However, those algorithms are not appropriate when the input image is underexposed or overexposed because they provide output images with a mean brightness close to the input and some results in a manner unsuitable for human perception.

Recently, image enhancement based on luminosity and contrast adjustment (LCA) [7], which augments HE, has been designed for a color retinal image. The first part of LCA creates a luminance gain matrix by correcting the gamma value of the value channel, $V$, in the hue-saturation-value (HSV) color space, and it uses the gain value to enhance the intensity of $R, G$, and $B$ channels. In the second part, the contrast is improved by converting the enhanced image to the $L^{*} a^{*} b$ color space and applying CLAHE [8] to adjust the local contrast of the luminance $L$ with the number of tiles and clip limit equal to $8 \times 8$ and 0.01 , respectively. LCA could handle the contrast enhancement, but neglects the color balance.

In 2016, Dai et al. [9] proposed retinal fundus image enhancement by using normalized convolution and noise removal (NCNR). The original image is used to extract the information in the background image by employing a normalized convolution filter [10]. The difference between the original image and the background image is then multiplied by a contrast factor and recombined with the original image in order to increase the image contrast. Finally, the fourth order PDEs [11] and a relaxed median filter [12] are applied to reduce noise in the image. Although this technique enhances detailed information by increasing the image contrast, especially in the vessel areas, the color balance of the output image is not proper for human perception and the NCNR method is time consuming.

The contrast enhancement methods, as described above, usually provide results in unnatural looks and unpleasing color balance. Motivated and inspired by the findings published in [13], Hubbard et al. analyzed brightness, contrast, and color balance of digital images compared to film retinal images in the AREDS reading center for the AMD screening. They divided 256 intensity levels into 16 scales, and defined the brightness at the peaks of each color channel to $12 / 16,6 / 16$, and $2 / 16$ for red $(R)$, green $(G)$, and blue $(B)$, respectively. Results in the color balance yielded at the band ratios of $G / R=0.5$ and $B / R=0.17$.

In this paper, we propose an improvement of the previously discussed methods to increase the visibility of a hidden anatomical structure in dark regions for non-uniform illumination retinal images. Gamma correction is firstly used to improve image luminosity. The image contrast is then enhanced by applying multi-scale tone manipulation, and finally, color balance is adjusted by specifying the image brightness based on Hubbard's model.

The performance of the proposed method was evaluated by using the publicly available datasets, the Diabetic Retinopathy Database (DIARETDB1) [14]. The 
DIARETDB1 dataset, acquired by Kauppi et al. [14], consists of 89 color retinal images. The images were taken with a $50^{\circ}$ field of view with the size of $1500 \times 1152$ pixels and stored in a 24-bit PNG format.

The results demonstrate that the proposed algorithm performs well in non-uniform illumination image enhancement. Images processed by our method have better visual quality and are better suited to human visual perception than those processed by NCNR and LCA. This will allow ophthalmologists to more visually and accurately diagnose the disease.

The paper proceeds as follows: the proposed method is described in Section 2, our experimental results appear in Section 3, and conclusions in Section 4.

\section{CONTRAST AND COLOR BALANCE ENHANCEMENT}

The proposed method consists of three modules (see Fig. 1): the Luminosity enhancement module, the Contrast and tone enhancement module, and the Color balance adjustment module. The luminosity enhancement module employs gamma correction for boosting up the visibility of anatomical details that were obscure in dark regions. In the second module, the Multi-scale tone manipulation is employed to improve the image contrast and tone. The color balance adjustment in the last module is performed to specify the brightness of red, green, and blue channel based on the Hubbard specification. A detailed description of each module is presented in the following subsections.

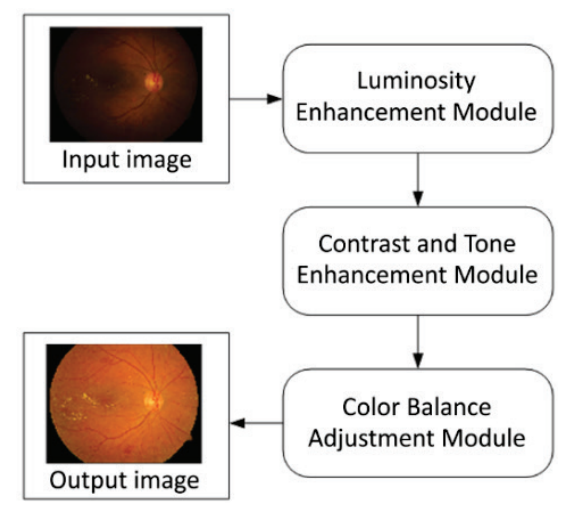

Figure 1 Framework of the proposed method.

\subsection{Luminosity Enhancement Module}

From our observation in the DIARETDB1 datasets, most images have some dark regions caused by uneven luminance. Those regions obscure the anatomical structures in the ocular fundus, and make the details necessary to make a diagnosis undetectable. To improve those dark regions of the image, the luminosity of the image needs to be enhanced. Mei et al. introduced a luminance gain matrix [7], which was derived from the gamma correction of the value channel in the HSV color space. The gain matrix showed good improvement in luminosity. The gain $G(x, y)$ could be rewritten in the simple form as:
$G(x, y)=\frac{m\left[\frac{v(x, y)}{m}\right]^{\frac{1}{2.2}}}{v(x, y)}$,

where $v$ refers to the luminance values from HSV, that is, $v(x, y)=\max (r(x, y), g(x, y), b(x, y))$ and $r(x, y), g(x, y)$, and $b(x, y)$ are intensity values at the coordinate $(x, y)$ from the $\mathrm{R}$, $\mathrm{G}$, and B channels, respectively. The parameter $m$ is the maximum value of $v$.

In particular, from our investigation in the DIARETDB1 dataset, the average dynamic range of red, green, and blue channels are [0-222], [0 - 157], and [0-78], respectively. Due to the dynamic range of the blue channel being narrow and its maximum intensity value being low, hence, the formula of $v(x, y)=\max (r(x, y), g(x, y), b(x, y))$ could be changed to $v(x, y)=\max (r(x, y), g(x, y))$ to reduce the computational time. Additionally, the parameter $m$ might be estimated from the maximum intensity of the red channel as $m=\max (r(x, y))$; in this case, the parameters $v$ and $m$ can be a parallel approximation.

The enhanced luminosity $R G B^{\prime}$, from the input image, $R G B$, can be obtained by multiplying pixel by pixel as in the following:

$R G B^{\prime}(x, y)=R G B(x, y) \cdot G(x, y)$
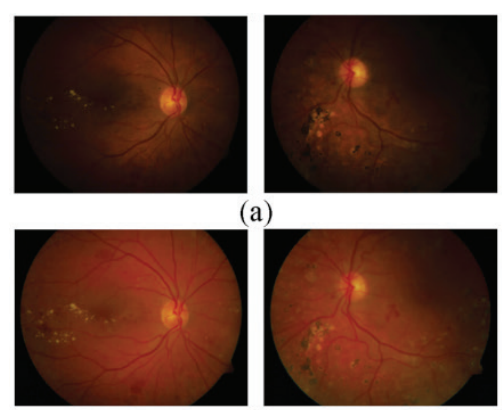

(b)

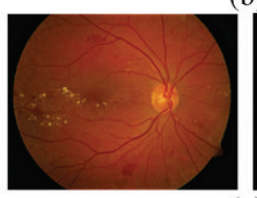

(

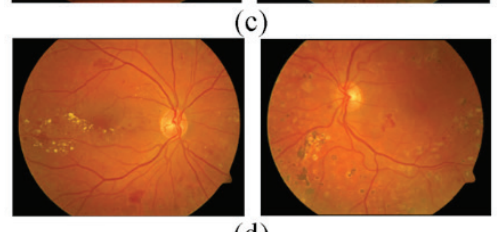

(d)
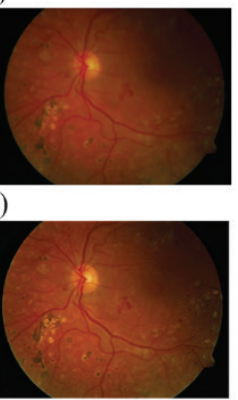

Figure 2 Results from each enhancement module: (a) Input images from the file Image007.png (left column) and Image027.png (right column); (b) Luminosity Enhancement; (c) Contrast and tone Enhancement; (d) Color balance adjustment.

The second row of Fig. 2 shows the enhanced luminosity as seen in the images; visibility is improved in the dark areas. The next step is to enhance the image contrast and tone to provide a better visibility of the retinal anatomical structures. 


\subsection{Contrast and Tone Enhancement Module}

Multi-scale tone manipulation is effectively used for improving the contrast and tone of images. In this module, the image is converted to the $L^{*} a^{*} b^{*}$ color space, while lightness, the $L$ component, is decomposed with different scales [15]. In our scheme, the decomposed image consists of a smooth base layer $\left(l_{1}\right)$ and two detail layers $\left(d_{0}\right.$ and $\left.d_{1}\right)$. Each layer is processed separately and recombined to generate the final result [15].

Farbman et al. [15] introduced a smooth layer, $l_{1}$, which can be obtained by using the WLS filter. When varying the parameter values of the WLS filter, the image tone is not smooth enough, especially, around the optic disk areas, and it results in an unnatural look as shown in Fig. 3(b). Hence, the WLS is replaced with the optic transfer function of Deeley et al. [16], and named as DOTF. The smoother lightness versions $l_{0}$ and $l_{1}$ can be defined as:

$l_{0}=F^{-1}\{F\{l\} \cdot D O T F\}$,

$l_{1}=F^{-1}\left\{F\left\{l_{0}\right\} \cdot D O T F\right\}$,

where $F$ and $F^{1}$ denote the Fourier transform and its inverse, and the formula of DOTF described in [16] is given as:

$$
D O T F=\exp \left[-\left(\frac{u}{(20.9-2.1 \Phi)^{(1.3-0.07 \Phi)}}\right)\right],
$$

where $u$ denotes the spatial frequency and $\Phi$ is the pupil diameter approximated to $4 \mathrm{~mm}$.

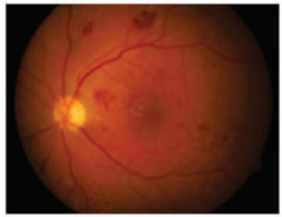

(a)

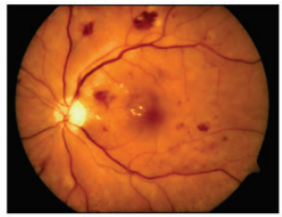

(b)

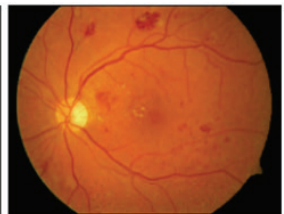

(c)
Figure 3 Comparison of results with a different filter: (a) Input image from the file Image008.png; (b) Output by using WLS; (c) Output by using OTF.

The lightness channel $l$ and two smoother lightness versions $l_{0}$ and $l_{1}$ are then used to generate two detail layers, $d_{0}$ and $d_{1}$, expressed in the form:

$d_{0}=S\left(\delta_{0}, l-l_{0}\right)$,

$d_{1}=S\left(\delta_{1}, l_{0}-l_{1}\right)$

where $S$ is a sigmoid curve which is defined as $S(a, x)=$ $1 /(1+\exp (-a x)) . \delta_{0}$ and $\delta_{1}$ are boosting factors for the $d_{0}$ and $d_{1}$ layers.

The base layer $l_{1}$ and two detail layers, $d_{0}$ and $d_{1}$ were then recomposed to form the enhanced lightness, $l^{\prime}$, which can be formulated as:

$l^{\prime}=\mu+S\left(\delta_{2}, \eta \cdot l_{1}-\mu\right)+d_{0}+d_{1}$, where $\eta$ denotes the exposure of the base layer, $\delta_{2}$ refers to the boosting factors for the base layer, and $\mu$ is the mean of the lightness range. We utilized parameters in agreement with those reported by Farbman et al. [15]. The parameters were $\delta_{0}=1, \delta_{1}=40$, and $\delta_{2}=1, \eta=1.0$ and $\mu=56$.

In the last step of this module, the contrast and tone of the enhanced image, $R G B^{\prime \prime}$, are obtained by replacing the $L$ component by $l^{\prime}$ and converting $L^{*} a^{*} b^{*}$ back to the RGB color space.

Fig. 4 shows the base layer and two detail layers corresponding to the image in Fig. 2 DOTF takes less processing time than WLS and gives a more natural look as shown in Fig. 3(c). The contrast and tone of the enhanced images in the third row of Fig. 2 have a better contrast when compared to the luminosity enhanced images in the second row. The next module is to adjust the image color balance.

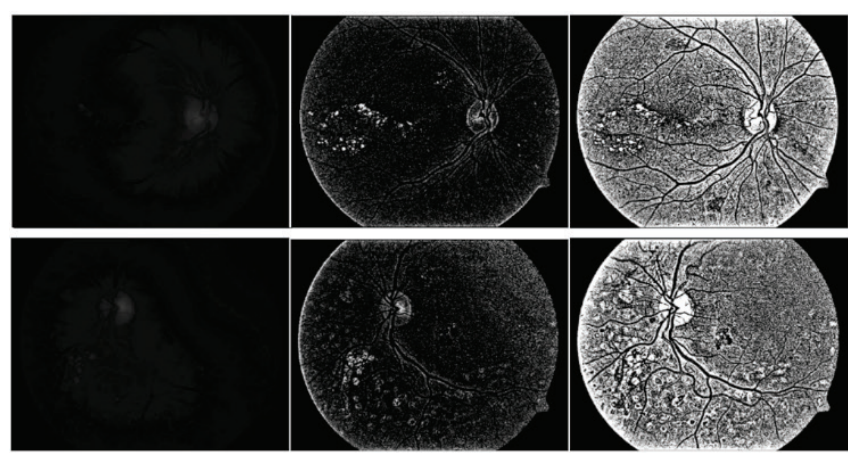

(a)

(b)

(c)

Figure 4 Corresponding base layer and two detail layers of the image in Fig. 2 : (a) Base layer $l_{1}$, (b) and (c) Detail layers $d_{0}$ and $d_{1}$.

\subsection{Color Balance Adjustment Module}

The findings by Hubbard et al. [13] lead to the main motivation for adjusting the color balance of color retinal images. The experimental results in the AREDS2 reading center confirm that a colored image with Hubbard's model parameters shows a greater contrast of drusen and pigment abnormalities compared to the normal retinal pigment epithelium. They specified the color balance with the color brightness of $R, G$, and $B$ to 192,96 , and 32, respectively, which resulted in color ratios of $G / R=0.5$ and $B / R=0.17$.

In this module, we use the specified brightness vector, $s b_{b}$ $=[192,96,32]$ from Hubbard's model to adjust the color balance of the enhanced result, $R G B^{\prime \prime}$, as processed in the above mentioned module. The color offset of the image $R G B^{\prime \prime}$ is adjusted by the difference between the brightness vector and the mean vector, $\mu$, of the $R G B^{\prime \prime}$ image, that is $\mu=\operatorname{mean}\left(R G B^{\prime \prime}\right)$.

The color balance for the final output image of $R G B^{\prime \prime \prime}$ is formulated as:

$R G B^{\prime \prime \prime}(x, y)=R G B^{\prime \prime}(x, y)+\left(s b_{b}-\mu\right)$,

The fourth row of Fig. 2 shows the results of the color balance adjusting module which give a greater contrast of the foreground compared to the background. 


\section{EXPERIMENTAL RESULTS}

In this section, the output results from our method are compared with NCNR [9] and LCA [7] by using the images from the DIARETDB1 dataset.

To evaluate the performance of our method, various quantitative metrics including measuring colorfulness $\left(M^{(3)}\right)$ [17], visual saliency-based index (VSI) [18], and lightness order error $(L O E)$ [19] are used to measure image quality. $M^{(3)}$ [17] is a colorfulness metric which is fitted to the perceptual data collected from a psychophysical experiment and it has a simple expression based on the opponent color. $V S I$ [18] measures the image quality in consistent with the human visual system or subjective evaluations. The method uses the visual saliency map as a feature to measure the image's local quality. $L O E[19]$ measures the lightness order error between the original and output image to assess naturalness preservation. The smaller the $L O E$ values, the better that naturalness is preserved. However, color image
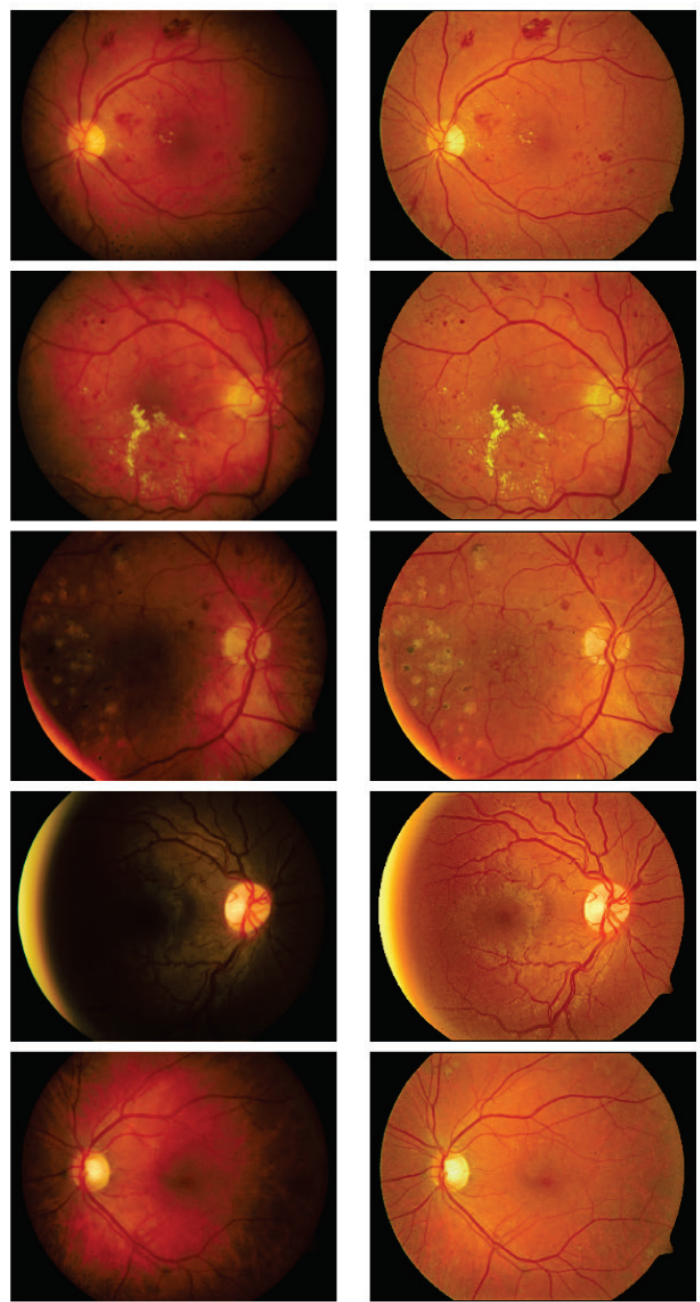

(a)

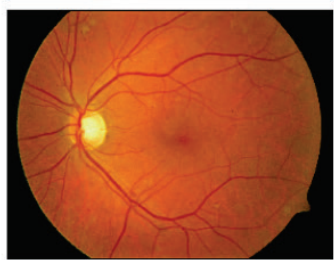

(b) assessment is difficult to evaluate by using only quantitative measurements. To determine if an image has good quality requires both quantitative and visual assessment. For the visual assessment, we visually inspected the output image to see if it retains a pleasing natural look and if lesions of the ocular diseases can be easily discriminated.

\subsection{Visual Assessment}

Visual assessments are shown in Fig. 5 with the images taking from DIARETDB1 where the first column represents the original images, and the output images consist of the proposed method in the second column, NCNR in the third column, and LCA in the fourth column. The output images, by employing the NCNR and LCA methods, generated dark color areas, especially for the blood vessel, macular, and the abnormal tissue such as hemorrhages, and small red blood dots.
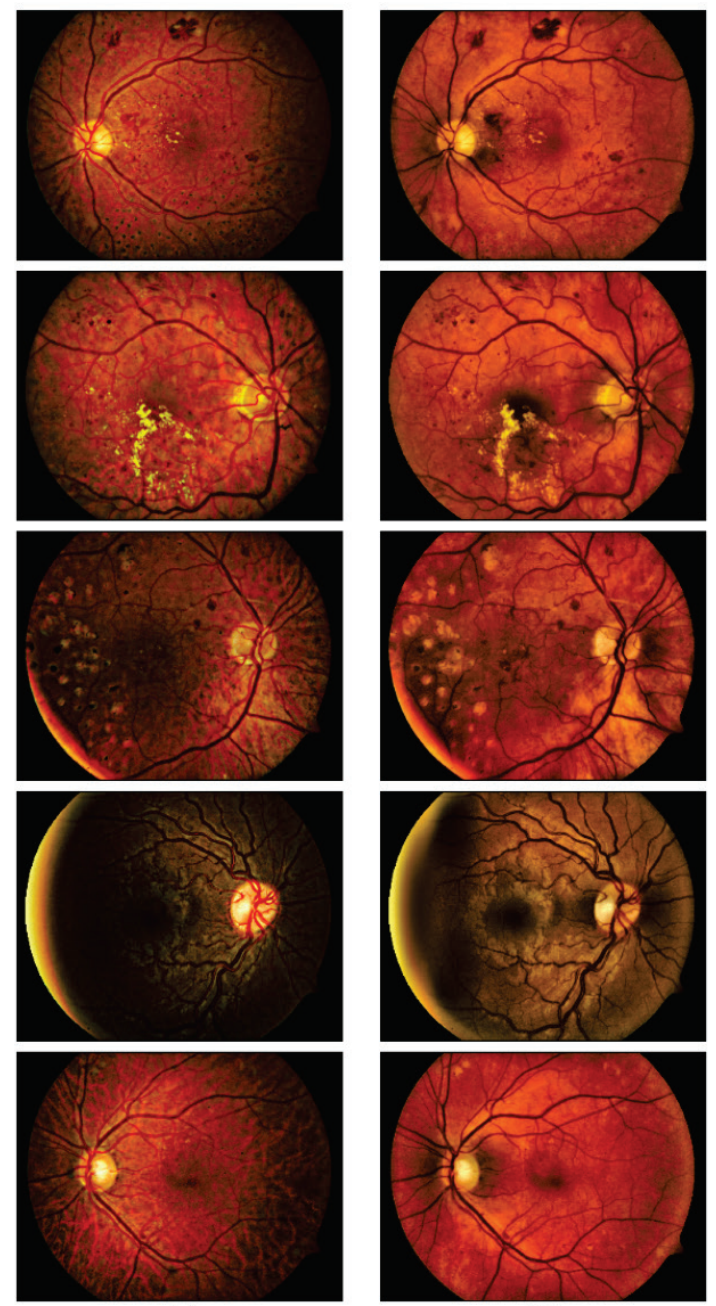

(c)

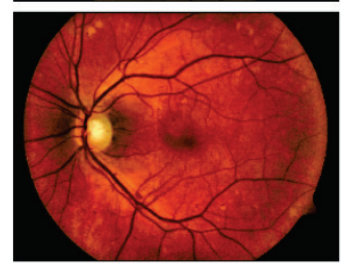

(d)

Figure 5 Results of different methods for the images: image008.png, image019.png, image029.png, image047.png, and image054.png from DIARETDB1: (a) Input images and output images, (b) the proposed method, (c) NCNR and (d) LCA.

Although the proposed method cannot perform image contrast as well as VCEA and LCA, our proposed method produces a pleasing visual appearance and maintains the color balance better than the other methods. The output results from the proposed method could reveal the hidden 
anatomical structures from the dark regions and may help ophthalmologists to improve their diagnostic accuracy.

Compared with the other methods, the proposed method enhances the contrast and tone of anatomical details. Additionally, it also produces a natural look with a proper color balance as specified by the reading center [13].

\subsection{Quantitative Assessment}

Tab. 1 shows all quantitative metric values, where each row corresponds to the enhancement methods and each column corresponds to the quantitative metrics in the form of an average and standard deviation value (Mean $\pm \mathrm{SD}$ ), calculated from 89 images from DIARETDB1. Illustrated are the mean and standard deviation of the color ratios, $M^{(3)}, V S I$, and $L O E$. From the experimental results in the column two and three of Tab. 1, the average green-to-red, and blue-to-red ratio of the output image from the proposed method were $0.50 \pm 0.00,0.17 \pm 0.00$, which meets the color balance specification from the Hubbard model. The proposed method gave the highest quantitative scores for $M^{(3)}(97.11 \pm 1.73)$ and VSI $(0.9574 \pm 0.0149)$. These highest scores imply that the proposed method yields better colorfulness and visual perception. The experimental results in the last column of Tab. 1 show that the proposed method scored the lowest for $L O E(97.39 \pm 35.15)$, and therefore can well preserve the naturalness.

Table 1 Performance of various methods on DIARETDB1

\begin{tabular}{|c|c|c|c|c|c|}
\hline Method & $G / R$ & $B / R$ & $M^{(3)}$ & VSI & $L O F$ \\
\hline Our method & $0.50 \pm 0.00$ & $0.17 \pm 0.00$ & $97.11 \pm 1.73$ & $0.9574 \pm 0.0149$ & $97.39 \pm 35.15$ \\
\hline NCNR & $0.39 \pm 0.06$ & $0.09 \pm 0.05$ & $72.23 \pm 19.27$ & $0.9539 \pm 0.0100$ & $134.77 \pm 29.17$ \\
\hline LCA & $0.39 \pm 0.06$ & $0.08 \pm 0.05$ & $86.47 \pm 18.57$ & $0.9556 \pm 0.0121$ & $474.66 \pm 116.02$ \\
\hline
\end{tabular}

\section{CONCLUSION}

In this paper, a new automatic image enhancement method is proposed to improve contrast and color balance for the non-uniform illumination retinal image. Our proposed method was tested on the DIARETDB1 dataset. The quantitative and visual assessment results demonstrate that the output images obtained by the proposed method give natural look and therefore are better suited for human visual perception in comparison to other methods. In terms of diagnoses made by ophthalmologists, the enhanced images obtained by our method could be used to assist ophthalmologists in the early detection of a disease and its diagnosis.

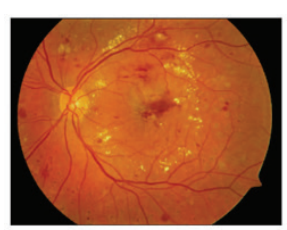

(a)

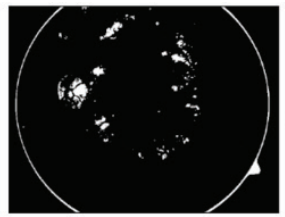

(b)

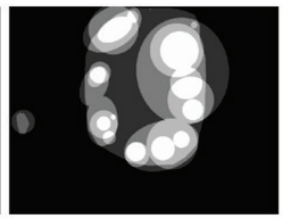

(c)
Figure 6 Example of a hard exudate detection: (a) Enhanced image of the image005.png; (b) Segmented result; (c) Hard exudate ground truth.

\section{FUTURE WORK}

Due to the output images derived from our proposed method they are standardized in color as shown in Fig. 5, hence, color features such as the color ratios: G/R and $B / R$ could be used to automatically detect the presence of abnormal lesions such as hard exudate, hemorrhage, and small red blood dot. Fig. 6 shows an example of hard exudate detection by thresholding only the $\mathrm{G} / \mathrm{R}$ color ratio feature. Fig. 6(b) depicts the hard exudate segment as a result from the enhanced input image in Fig. 6(a). To increase the accuracy of the detection, the optic disk area should be removed from the retinal color image before detection and an effective SVM classification should be applied to classify the hard exudate instead of using the threshold; the classification results are shown in Fig. 6(c).

\section{Conflicts of Interest}

The authors declare that there is no conflict of interest regarding the publication of this paper.

\section{Acknowledgments}

This work was supported by the Prince of Songkla University Grant no. SCI600372S.

\section{Remark}

The article was orally presented at the $23^{\text {rd }}$ International Computer Science and Engineering Conference (ICSEC2019).

\section{REFERENCES}

[1] Main causes of visual impairment. WHO. Retrieved from http://who.int/blindness/causes/priority/en

[2] Wong, W. L., Su, X., Li, X., \& Cheung, C. M. (2014). Global prevalence of age-related macular degeneration and disease burden projection for 2020 and 2040: a systematic review and meta-analysis. The Lancet Global Health, 2(2), 106-116. https://doi.org/10.1016/S2214-109X(13)70145-1.

[3] Abramoff, M. D., Garvin, M. K., \& Sonka, M. (2010). Retinal imaging and image analysis. IEEE Rev in Biomed Eng, 3, 169208. https://doi.org/10.1109/RBME.2010.2084567

[4] Wang, C., \& Ye, Z. (2005). Brightness preserving histogram equalization with maximum entropy: a variational perspective. IEEE Trans. Consum. Electron., 51(4), 1326-1334. https://doi.org/10.1109/TCE.2005.1561863

[5] Ibrahim, H. \& Kong, N. (2007). Brightness preserving dynamic histogram equalization for image contrast enhancement. IEEE Trans. Consum. Electron., 53, 1752-1758. https://doi.org/10.1109/TCE.2007.4429280 
[6] Sheet, D., Garud, H., Suveer, A., Mahadevappa, M., \& Chatterjee, J. (2010). Brightness preserving dynamic fuzzy histogram equalization. IEEE Trans. Consum. Electron, 56(4), 2475-2480. https://doi.org/10.1109/TCE.2010.5681130

[7] Mei, Z., Kai, J., Shaoze, W., Juan, Y., \& Dahong, Q. (2017). Color retinal image enhancement based on luminosity and contrast adjustment. IEEE Rev. Biomed. Eng.(99), 1-7. https://doi.org/10.1109/TBME.2017.2700627

[8] Zuiderveld, K. (1994). Contrast limited adaptive histogram equalization. San Diego: Academic press professional, 474485. https://doi.org/10.1109//CACCI.2014.6968381

[9] Peishan, D., Hanwei, S., Jianmei, Z., Ling, L., Jing, W., \& Min, F. (2016). Retinal fundus image enhancement using the normalized convolution and noise removing. International Journal of Biomedical Imaging, 1-13. https://doi.org/10.1155/2016/5075612

[10] Gastal, E. S., \& Oliveira, M. M. (2011). Domain transform for edge-aware image and video processing. ACM Trans. on Graphics, 30(4), 1244-1259. https://doi.org/10.1145/2010324.1964964

[11] Rajan, J., Kannan, K., \& Kaimal, M. R. (2008). An improved hybrid model for molecular image denoising. J. of Math. Imaging and Vision, 31(1), 73-79. https://doi.org/10.1007/s10851-008-0067-4

[12] Hamza, A. B., Luque-Escamilla, P. L., Martınez-Aroza, J., \& Roman-Roldan, R. (1999). Removing noise and preserving details with relaxed median filters. J. of Math. Imaging and Vision, 11(2), 161-177. https://doi.org/10.1023/A:100839551

[13] Hubbard, L. D., Danis, R. P., Neider, M. W., Thayer, H. D., Wabers, H. D., White, J. K., . . . Pugliese, M. F. (2008). Brightness, contrast, and color balance of digital versus film retinal Images in the age-related eye disease study 2. Journal of Investigative Ophthalmology \& Visual Science, 49(8), 32693282. https://doi.org/10.1167/iovs.07-1267

[14] Tomi, K., Valentina, K., Kristian, K. J., Lasse, L., \& Liris, S. (2007). Diaretdb1 diabetic retinopathy database and evaluation protocol. in Proc. of the British Machine Vision Conference. University of Warwick, UK, 252-261. https://doi.org/10.5244/C.21.15

[15] Farbman, z., Fattal, R., Lischinski, D., \& Szeliski, R. (2008). Edge-preserving decompositions for multi-scale tone and detail manipulation. ACM TOG, 27(3), 67. https://doi.org/10.1145/1360612.1360666

[16] Deeley, R. J., Drasdo, N., \& Charman, N. W. (1991). A simple parametric model of the human ocular modulation transfer function. Phthalmic \& Physiological Optics, 11(1), 91-93. https://doi.org/10.1111/j.1475-1313.1991.tb00200.x

[17] Hasler, D. \& Susstrunk, S. (2003). Measuring colorfulness in natural images. Proc. SPIE, 5007, 87-95. https://doi.org/10.1117/12.477378

[18] Zhang, L., Shen, Y., \& Li, H. (2014). VSI: A visual saliencyinduced index for perceptual image quality assessment. IEEE Trans Image Process, 23(10), 4270-4281. https://doi.org/10.1109/TIP.2014.2346028

[19] Wang, S., Zheng, J., Hu, H., \& Li, B. (2013). Naturalness preserved enhancement algorithm for non-uniform illumination images. Trans. Image Process., 22(9), 60-70. https://doi.org/10.1109/TIP.2013.2261309
Authors' contacts:

Preecha VONGHIRANDECHA

Prince of Songkla University,

15 Kanjanavanich, 90110, Hatyai, Songkhla, Thailand

preecha.v@psu.ac.th

Montri KARNJANADECHA, PhD

Prince of Songkla University,

15 Kanjanavanich, 90110, Hatyai, Songkhla, Thailand montri@coe.psu.ac.th

Sathit INTAG, PhD

Prince of Songkla University, 15 Kanjanavanich, 90110, Hatyai, Songkhla, Thailand sathit.@@su.ac.th 